Revue des patrimoines

Le patrimoine religieuX des $\mathrm{XIX}^{\mathrm{e}}$ et $\mathrm{XX}^{\mathrm{e}}$ siècles

\title{
Le couvent de la Visitation Sainte-Marie de Fourvière (Lyon, Rhône)
}

\section{Catherine Guégan}

\section{(2) OpenEdition}

Journals

Édition électronique

URL : http://journals.openedition.org/insitu/5620

DOI : 10.4000/insitu. 5620

ISSN : 1630-7305

\section{Éditeur}

Ministère de la culture

Référence électronique

Catherine Guégan, « Le couvent de la Visitation Sainte-Marie de Fourvière (Lyon, Rhône) », In Situ [En ligne], 11 | 2009, mis en ligne le 18 avril 2012, consulté le 19 avril 2019. URL : http:// journals.openedition.org/insitu/5620; DOI : 10.4000/insitu.5620

Ce document a été généré automatiquement le 19 avril 2019

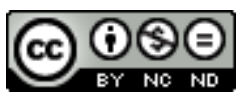

In Situ Revues des patrimoines est mis à disposition selon les termes de la licence Creative Commons Attribution - Pas d'Utilisation Commerciale - Pas de Modification 4.0 International. 


\section{Le couvent de la Visitation Sainte-Marie de Fourvière (Lyon, Rhône)}

\section{Catherine Guégan}

Figure 1

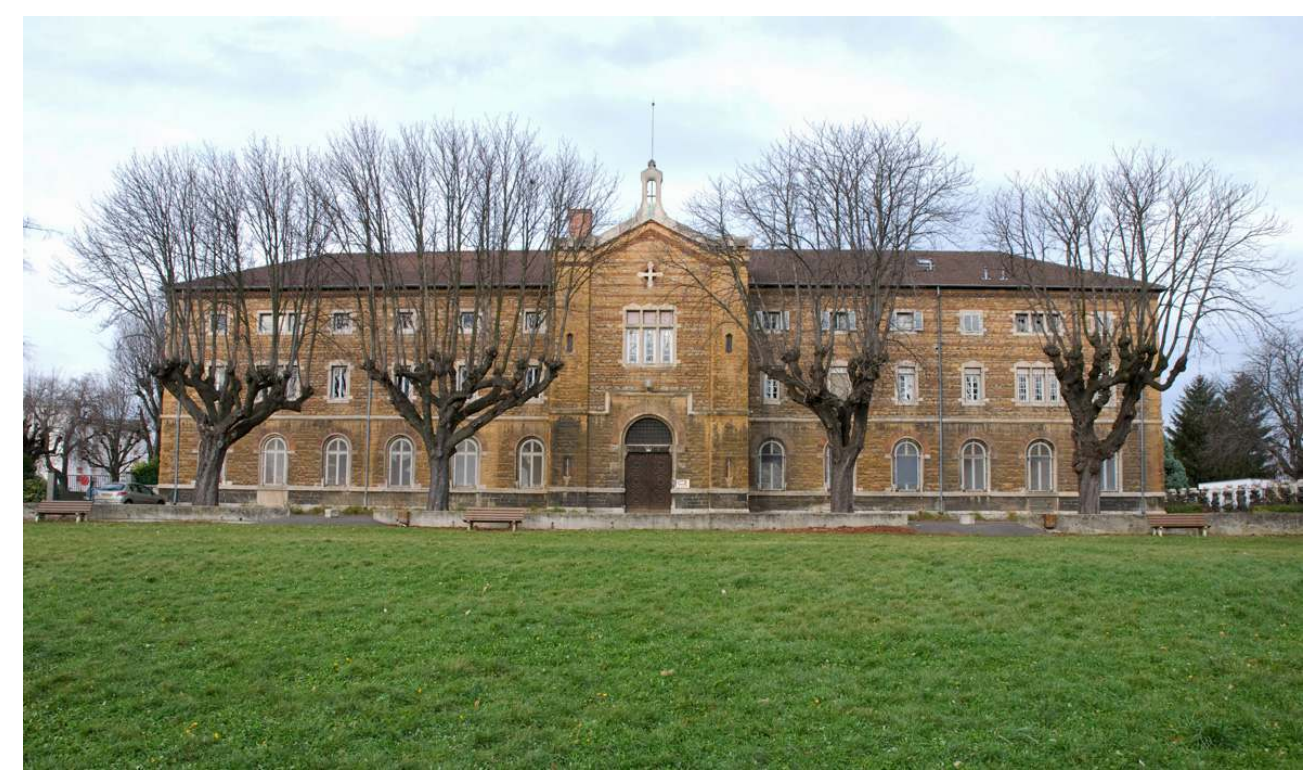

Le couvent de la Visitation Sainte-Marie de Fourvière, Lyon (Rhône). Élévation principale

Phot. C. Guégan, 2007. @ Inventaire du patrimoine culturel, Région Rhône-Alpes/Ville de Lyon, ADAGP

1 Construit en 1854 sur la colline de Fourvière qui abritait alors de nombreux monastères, en retrait des berges de la Saône, le couvent de la Visitation Sainte-Marie ${ }^{1}\left(\right.$ fig. $^{\circ} \mathbf{n}^{\circ}$ ) est l'une des premières œuvres lyonnaises de Pierre-Marie Bossan (1814-1888), plus connu pour être l'architecte de la basilique Notre-Dame de Fourvière. À cette date, Bossan a déjà à son actif quelques réalisations dans le domaine de l'architecture religieuse, dont l'église néo-gothique Saint-Georges (Lyon 5e, 1844), qu'il qualifiera plus tard d' «erreur de 
jeunesse ». Un voyage en Italie entre 1845 et 1850 lui fait en effet découvrir l'art roman et surtout byzantin, dont l'influence va être déterminante dans ses créations ultérieures, modifiant en profondeur sa conception de l'architecture. Le couvent de la Visitation de Fourvière en porte à la fois la marque et en constitue la première formulation.

Comme beaucoup d'autres, le monastère a connu au XXe siècle les vicissitudes liées à la baisse des vocations et aux difficultés économiques de l'ordre, qui sont allées s'accentuant après la Seconde guerre mondiale. Vendu à la Ville de Lyon en 1965, il abrite depuis 1974, par l'effet d'un échange de terrains, le service central des archives des Hospices civils de Lyon. Or ces derniers ont entrepris, depuis le début des années 2000, de rationaliser la gestion de leurs activités hospitalières et de leur patrimoine immobilier. La réorganisation et le regroupement des services se sont opérés soit par transfert vers de nouveaux lieux (hôpital mère-enfant de Bron) soit par réaménagement des structures existantes (hôpital Édouard-Herriot). Certains bâtiments jugés vétustes ont été cédés (l'Antiquaille) ou fermés (Debrousse). C'est dans ce cadre que s'inscrit le projet de vente du site de Fourvière, qui occupe la quasi-totalité de la surface de l'ancien monastère, vaste réserve foncière entourée d'espaces verts qui en font un îlot de calme à l'écart de la ville, propre à susciter l'intérêt des investisseurs. La question de sa reconversion étant posée, il paraissait important de mener une enquête approfondie sur cet édifice encore peu connu ${ }^{2}$, et ce d'autant plus qu'il ne bénéficie pour l'heure, comme beaucoup d'autres œuvres de Bossan, d'aucune protection au titre des monuments historiques. Ce sont les principaux éléments de cette étude qui sont ici transcrits, visant à mettre en valeur les parties de l'œuvre qui mériteraient d'être préservées, voire restaurées.

\section{Installation des visitandines à Fourvière}




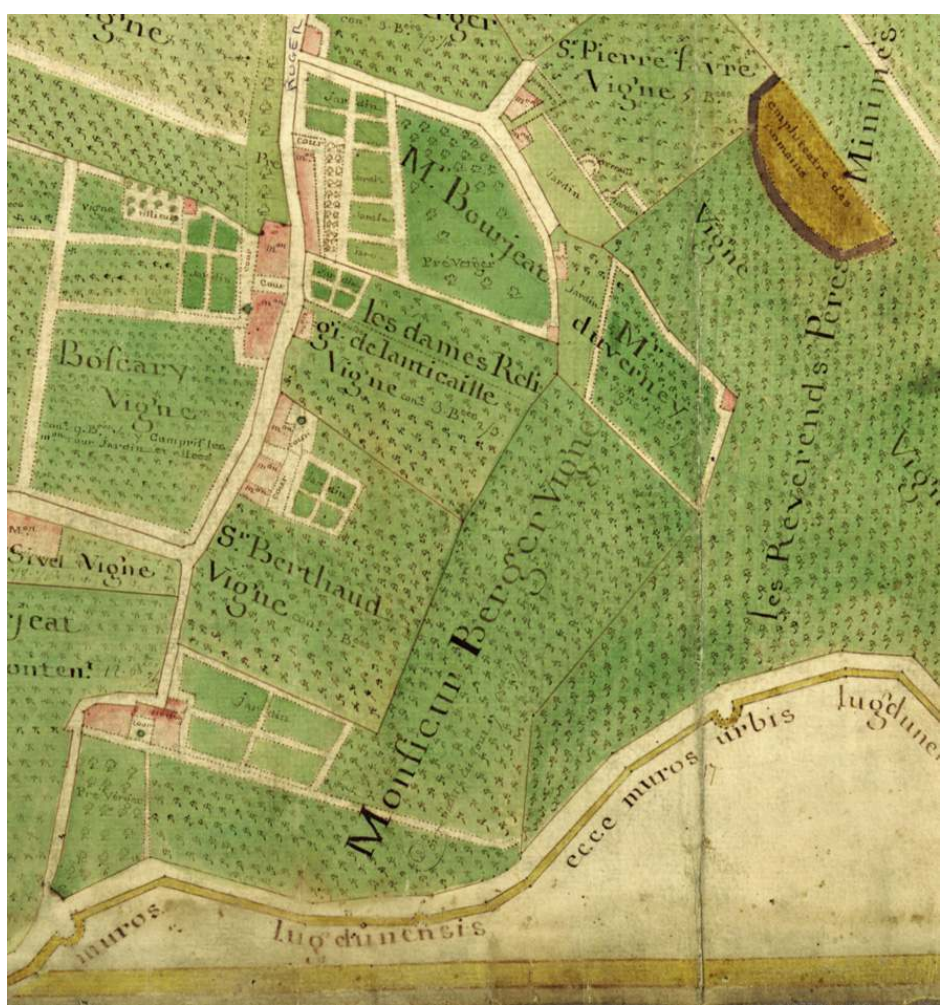

Plan des quartiers de Fourvière et Saint-Just par Chavallard. 1 :300. 1767. AC Lyon. 2 S 57 (détail)

Phot. J. Gastineau, 2003. (c) AM Lyon

Depuis leur retour à Lyon au début du XIX siècle, les visitandines, autrefois installées dans les couvents de Bellecour, de l'Antiquaille et de la rue des Farges, s'étaient établies sur la colline de la Croix-Rousse, rue du Chapeau rouge. Mais gênées par la densité démographique du quartier et échaudées par l'insurrection des Canuts et les remous de la Révolution de 1848, elles songèrent dès cette date à s'installer sur la colline de Fourvière. C'est ainsi qu'elles acquirent en 1850, conjointement avec les carmélites, une propriété sise sur la partie occidentale de la colline, dont la position dominante mais en retrait des pentes leur permettait de jouir d'une vue exceptionnelle sur la ville tout en étant totalement dissimulées des regards extérieurs. S'étendant sur plus de 3 hectares et close de murs, la propriété comportait plusieurs corps de bâtiments d'habitation et d'exploitation. Longtemps occupé par des vignes et des vergers, le site conservait au milieu du XIX siècle l'aspect rural qu'il avait au siècle précédent (fig. $\mathbf{n}^{\circ}$ 2). Aux visitandines fut attribuée la partie occidentale de la propriété, vierge de bâtiments mais déclinant rapidement vers le sud, où elle était bordée par le chemin des remparts du fort de Saint-Just, ainsi qu'une petite maison à l'ouest du terrain des carmélites, d'où elles pourraient surveiller l'érection de leur nouveau couvent. Ce dernier devait être construit sur la partie nord de la parcelle, où le terrain était relativement plat. Avant d'entreprendre les travaux, les religieuses acquirent en 1853 un deuxième terrain au sudest de leur parcelle, appartenant aux Dames de la Compassion. C'est là une démarche qui guidera leur politique foncière et immobilière tout au long du XIX siècle. Elles n'auront de cesse en effet d'agrandir leur propriété et d'édifier de nouveaux bâtiments pour y loger pensionnaires et aumônier", afin d'affecter le bâtiment principal à leur seul usage, ce que, pour des raisons d'économie, elles n'avaient pu faire dans un premier temps. 
Pour réaliser leur projet, elles firent appel à Pierre-Marie Bossan (1814-1888) dont la sœur, Marie-Aimée, était entrée au monastère en 1852; il s'agit d'une des premières œuvres lyonnaises de l'architecte après son retour d'Italie, vers 1850. Le choix de Bossan est à porter au crédit de la supérieure de l'ordre, Mère Marie-Régis Deville, qui joua un rôle important dans la définition du projet architectural. Elle souhaitait en effet un bâtiment peu onéreux, en raison de la somme relativement modique dont elle disposait ${ }^{5}$, et respectant le plan-type du Coutumier de l'ordre pour la disposition espaces. Ce plan aux proportions harmonieuses et régulières, repose sur un rigoureux principe de symétrie. Pour évoquer le calme monastique, en traduire l'équilibre et la plénitude, l'architecte choisit de prendre le carré pour module, utilisé tant pour les espaces conventuels que pour l'église.

\section{La construction du couvent}

Dès son retour en France, Bossan a très rapidement sous-traité ses chantiers à des collaborateurs, notamment en raison de sa foisonnante production de dessins pour les arts liturgiques. Incapable de mettre ses plans au propre et d'établir des devis exacts, il avait recours à eux pour rendre ses idées à partir de petits croquis jetés sur le papier. $\mathrm{Ne}$ voulant avoir aucune relation avec l'administration ou les maîtres d'ouvrage, il leur en déléguait la charge, leur confiant également le suivi de l'exécution des travaux ${ }^{6}$. Enfin, installé à La Ciotat depuis 1845 , d'où il était parti pour l'Italie, il répugnait à se rendre à Lyon et ne s'y déplaçait que de façon exceptionnelle.

6 Les travaux débutèrent en 1853 par l'érection du mur de clôture, après que les visitandines eurent acquis le terrain des Dames de la Compassion ${ }^{7}$ et les deux pavillons du télégraphe de Chappe qui surplombaient leur propriété 8 . Ils se poursuivirent l'année suivante avec la construction du couvent, malgré l'absence de fonds permettant d'en financer la totalité. . L'exécution en fut confiée aux entrepreneurs Parot et Boudet et au charpentier Clément Martinant ${ }^{10}$, et peut-être dirigée par l'architecte Charles Franchet ${ }^{11}$. Les Annales du couvent mentionnent cependant la présence d'un autre architecte, Guillaume Léo, aux côtés de Bossan ${ }^{12}$. Tous deux sont de fait des collaborateurs réguliers de Bossan dans les années 1850-60 ${ }^{13}$. Le 6 juin 1854, Mgr Franzoni, archevêque de Turin en exil, posait la première pierre au nom du cardinal de Bonald. Quatre sœurs furent chargées de suivre le chantier et s'installèrent en mai 1855 sur le site de Fourvière, dans le petit bâtiment mitoyen du Carmel déjà mentionné, appelé « maison du repassage ». De fait, lorsque les visitandines quittèrent la Croix-Rousse pour s'installer à Fourvière le 16 juin $1856^{14}$, les travaux de l'église et de l'aile nord, à l'angle de laquelle s'inscrivent les deux sacristies, n'étaient pas complètement achevés. D'après les sources, ils furent couverts peu avant le 24 octobre 1857, date de la cérémonie de prise d'habit d'une sœur. L'église et le chœur des religieuses avaient été bénis un peu plus tôt, le 26 septembre, en même temps qu'était dite la première messe ${ }^{15}$. À cette date, le gros œuvre était donc achevé, mais les travaux se poursuivirent dans l'église jusqu'en 1865.

7 En l'absence de plans d'origine, il est difficile de restituer la distribution des espaces intérieurs, le bâtiment ayant été profondément remanié au moment de sa transformation en dépôt d'archives (fig. $\mathbf{n}^{\circ} \mathbf{3}$ ). Les sources sont donc essentielles pour en reconstituer la disposition originelle. Établi à partir du plan modèle figurant dans le Coutumier de l'ordre, auquel Bossan avait été tenu, on l'a vu, de se conformer, le plan de Visitation de Fourvière en reprend les principales caractéristiques ${ }^{16}$. De plan carré, le bâtiment 
s'organise autour d'un cloître (fig. $\left.\mathbf{n}^{\circ} \mathbf{4}\right)$ fermé sur un côté par l'église. Le chœur des religieuses, perpendiculaire à l'église, s'élevait à l'origine sur les trois niveaux de l'édifice et était éclairé de hautes baies, en parties murées aujourd'hui. L'ouverture sur le chœur de l'église, vaste arcade en plein cintre supportée par deux colonnettes de stuc dont il était séparé par une grille de fer forgé reposant sur une balustrade de pierre, est aujourd'hui également murée, mais le soubassement de pierre est toujours en place. Deux sacristies jouxtent l'église dans l'angle nord-ouest du couvent. L'une destinée au prêtre desservant, accessible uniquement depuis l'extérieur par une porte-fenêtre (dont la partie basse a été murée), l'autre réservée aux religieuses, à laquelle on accédait depuis le cloître. Les sacristies étaient séparées par un mur dans lequel étaient pratiquées deux ouvertures. Dans la première baie se trouvait un confessionnal, toujours en place; dans la seconde, un bloc de pierre, aujourd'hui déposé dans le jardin du gymnase, supportait une cloison de bois dans laquelle était ménagé un tour pour le passage des vases sacrés. Le mur entre les deux sacristies était orné de sentences peintes, toujours visibles : « Je suis le pain vivant qu [i est descendu] du ciel $»^{17}$ et "Mes délices sont d'être avec les enfants des hommes $»^{18}$. Dans la sacristie extérieure, on peut voir la trace d'un escalier qui communiquait avec le premier étage, où se trouvait une petite pièce initialement destinée au desservant.

Figure 3

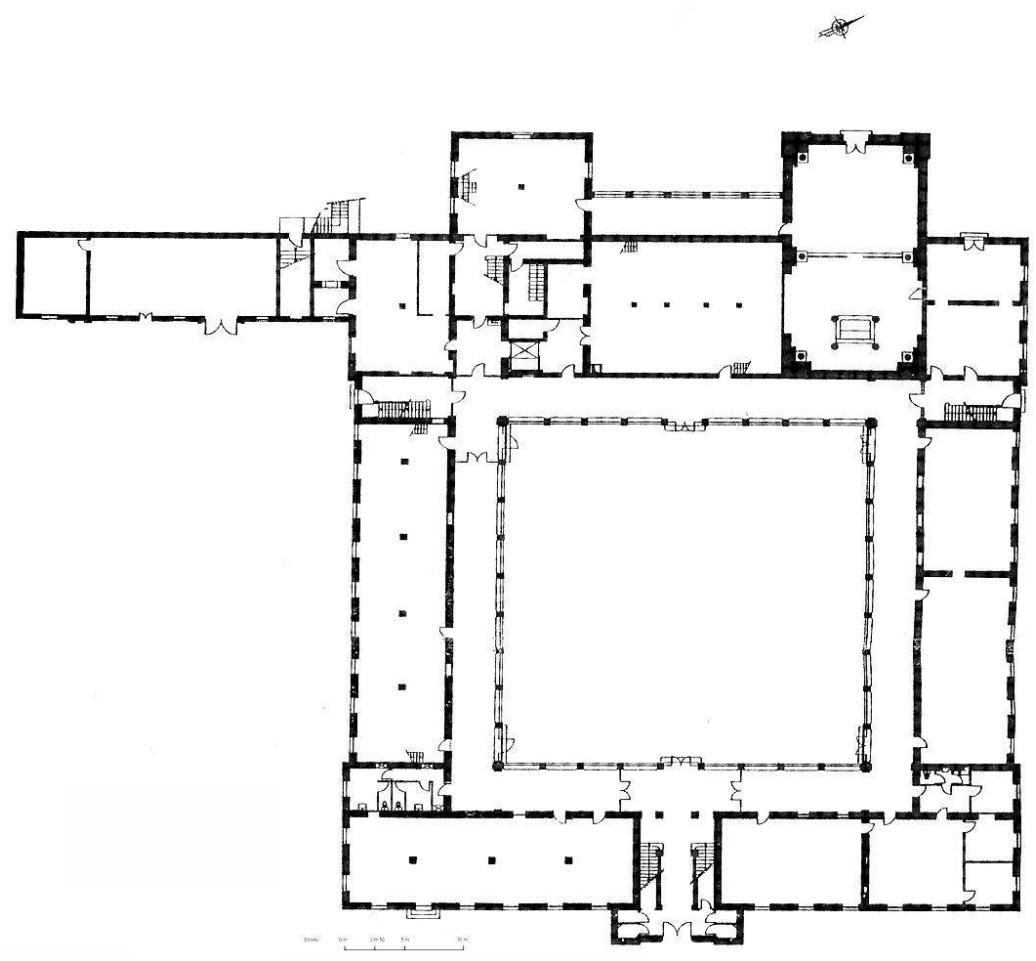

Plan du rez-de-chaussée, état actuel

(c) Hospices civils de Lyon, 1999 
Figure 4

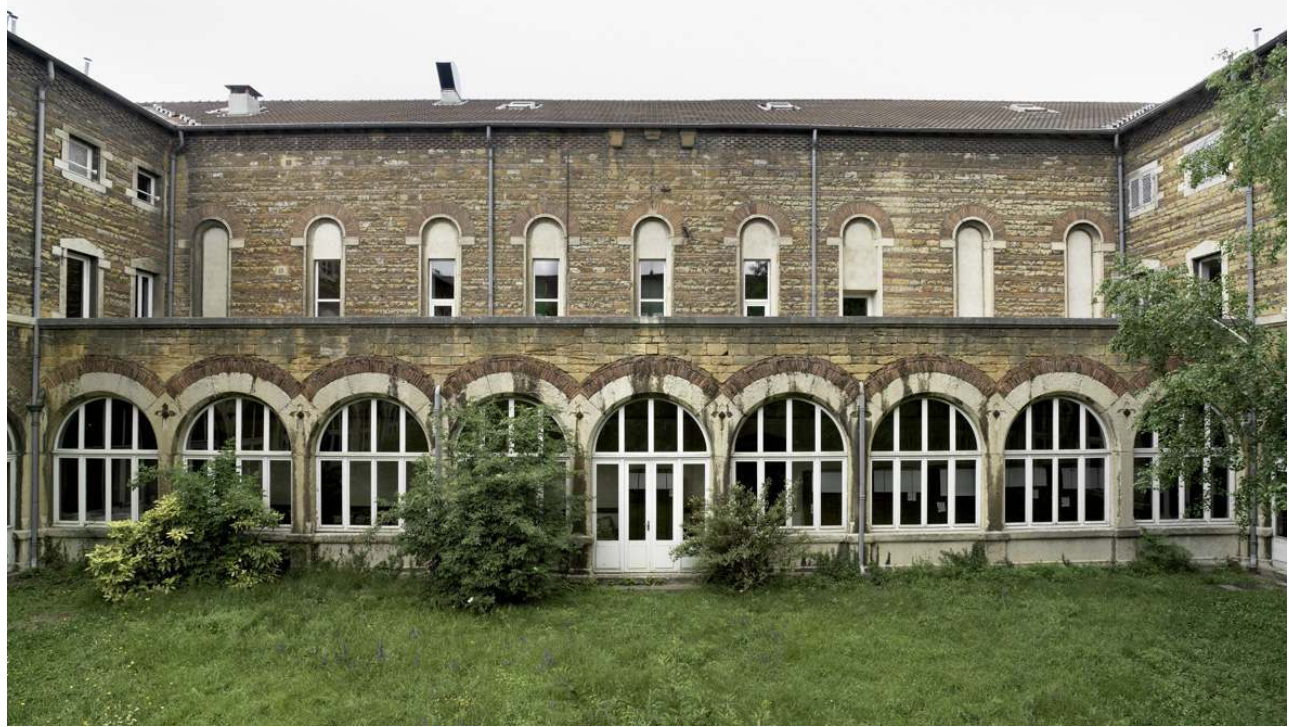

Cloître, élévation est

Phot. É. Dessert, 2007. @ Inventaire du patrimoine culturel, Région Rhône-Alpes/Ville de Lyon, ADAGP

8 Située dans l'aile est, l'entrée principale ouvre sur un vestibule donnant sur les galeries du cloître.

Figure 5

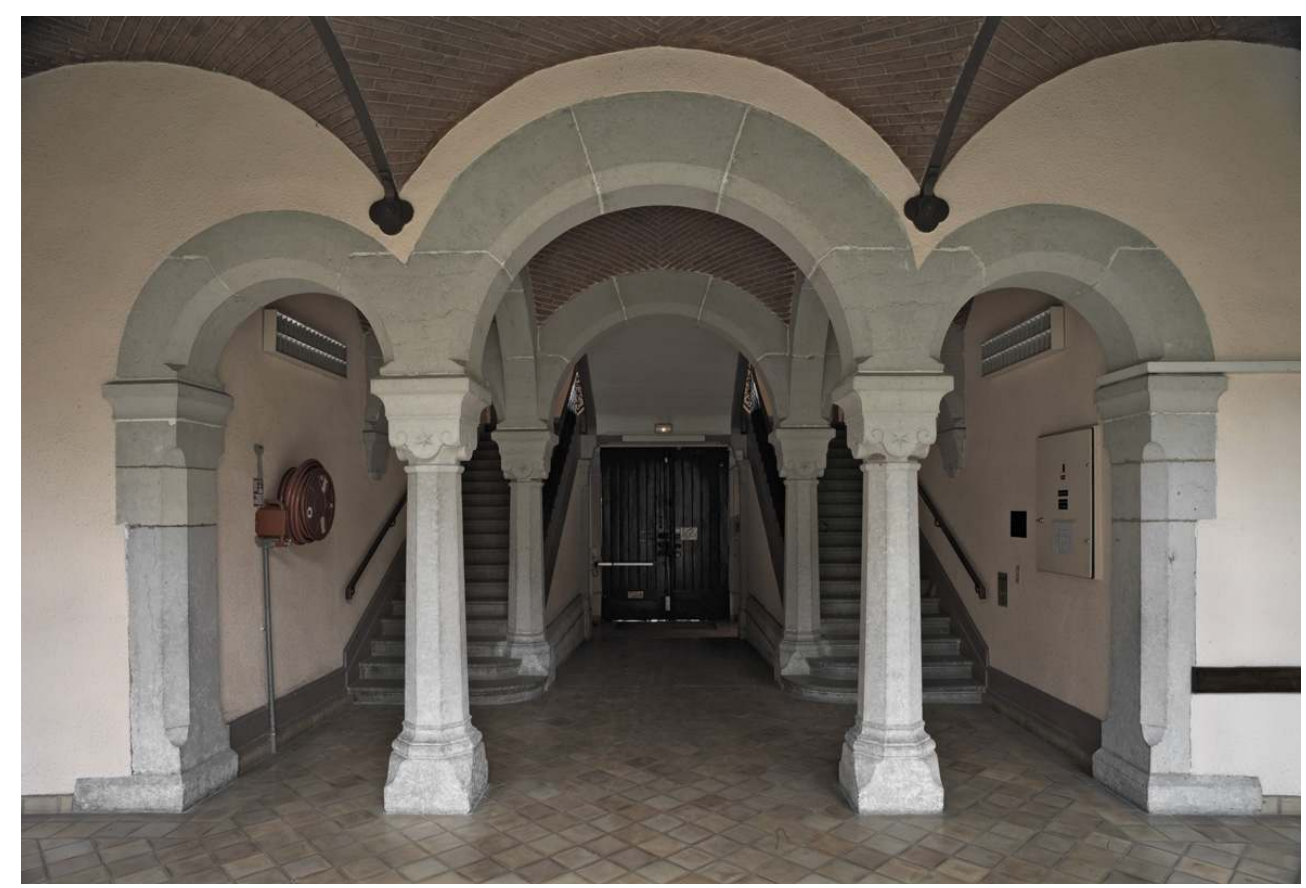

Vue du vestibule et de l'escalier principal, depuis le cloître

Phot. É. Dessert, 2008. @ Inventaire du patrimoine culturel, Région Rhône-Alpes/Ville de Lyon, ADAGP

9 Faisant face au cloître, un escalier à volée double (fig. $\mathbf{n}^{\circ}$ 5) conduit aux étages, également desservis par deux autres escaliers symétriquement positionnés aux angles nord et sud du couvent. L'escalier sud desservait l'aile du pensionnat, l'escalier nord permettait un accès 
direct à l'infirmerie. Un quatrième escalier, auquel on pouvait accéder de l'extérieur par une entrée aujourd'hui fermée (vraisemblablement l'entrée du parloir), située dans l'avant-corps sud de l'élévation postérieure, desservait les ailes sud et ouest. Cette même entrée donnait accès à la galerie qui longe le chœur des religieuses sur l'élévation postérieure du couvent. Sa fonction n'est pas très claire : peut-être abritait-elle le parloir, à moins qu'elle n'ait servi de passage intérieur pour rejoindre la nef de l'église, sur laquelle ouvre une petite porte.

10 Comme dans les autres monastères de l'ordre, le rez-de-chaussée abritait les pièces liées à la vie commune, difficiles à localiser aujourd'hui : la cuisine, le réfectoire, la lingerie, le cabinet des ouvrages, celui des papiers, le noviciat, la roberie, l'économat et le cabinet de la supérieure ${ }^{19}$. Dans l'aile ouest, l'église, le chœur des religieuses, qui faisait également office de salle de communauté, les parloirs, à proximité de la chambre des tourières qui, selon le plan du Coutumier, devait se situer près de la chapelle. Afin de maintenir une nette séparation des espaces entre lieux conventuels et lieux d'enseignement, le pensionnat était logé dans l'aile sud du bâtiment, à l'exclusion sans doute du rez-dechaussée ; en raison du coût de la construction, les religieuses avaient en effet renoncé à faire élever un bâtiment indépendant ${ }^{20}$.

11 La distribution des pièces évolua conjointement aux agrandissements du couvent et à l'adjonction de nouveaux bâtiments. En 1887, le noviciat et la roberie furent déplacés au second étage afin de créer une salle du chapitre, qui vint remplacer l'économat et la roberie. En 1892 intervinrent de nouvelles modifications : le noviciat fut une nouvelle fois déplacé et remplacé par une bibliothèque, l'économat fut implanté dans trois appartements du pensionnat (salle Louis de Gonzague, cabinet de physique (dont les meubles furent installés à l'étage de l'aumônerie) et réfectoire ${ }^{21}$; une cave à légumes fut également creusée ${ }^{22}$. Enfin, l'une des deux sacristies fut utilisée comme salle d'archives ${ }^{23}$. Ces modifications, et l'empiètement des pièces du couvent sur l'aile du pensionnat corroborent l'hypothèse que le «bâtiment de la galerie » construit en 1890 par SainteMarie Perrin (1835-1917) en fond de parcelle, au nord du terrain, était destiné à loger les pensionnaires au premier étage, dans des chambres individuelles et non plus dans des dortoirs.

Les étages du couvent reprennent la même distribution des espaces entre pensionnaires et religieuses : les cellules de ces dernières sont réparties dans les ailes est, nord et ouest de part et d'autre d'un large couloir. L'infirmerie était installée dans l'aile nord, près de l'église ; les sœurs malades pouvaient même assister à l'office depuis une pièce dont les ouvertures donnaient sur le chœur de cette dernière. Les pensionnaires étaient logées dans l'aile sud, où se trouvaient des salles de classe et de travail ainsi que leur réfectoire; les dortoirs étaient au deuxième étage. Elles avaient leur propre chapelle, appelée " chapelle des enfants de Marie » ou " tribune des élèves " ${ }^{24}$, d'où elles pouvaient assister aux offices. La pièce surplombait en effet le chœur des religieuses, sur lequel elle ouvrait. De cette tribune, on avait également vue, quand la grille était ouverte, sur le chœur de l'église, grâce à "six grandes fenêtres successives et cintrées " (aujourd'hui murées et non visibles ${ }^{25}$ ). On y accédait par le grand escalier au décor de faux appareil de pierre de l'aile ouest. Une terrasse, longeant le chœur des religieuses au premier étage, côté cloître, peut-être accessible aux pensionnaires, relie les ailes sud et nord; elle permettait aux malades de s'aérer sans avoir à rejoindre le rez-de-chaussée. Très logiquement, le gymnase viendra plus tard s'inscrire à proximité du pensionnat, perpendiculairement à 
l'aile sud, toujours dans le souci de séparer la clôture du monastère de ses activités pédagogiques et éducatives.

\section{Figure 6}

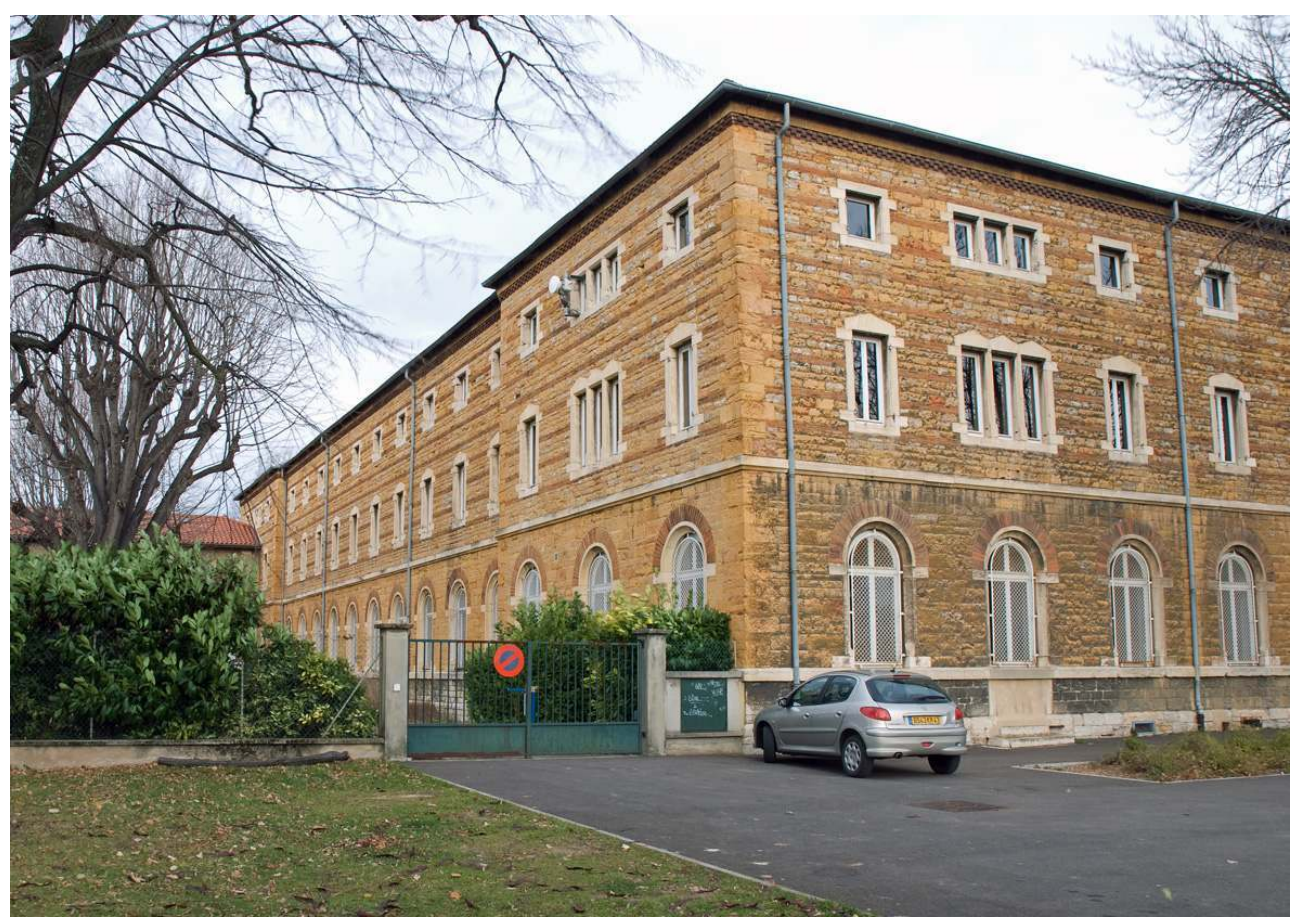

Élévation principale et latérale sud

Phot. C. Guégan, 2007. @ Inventaire du patrimoine culturel, Région Rhône-Alpes/Ville de Lyon, ADAGP 


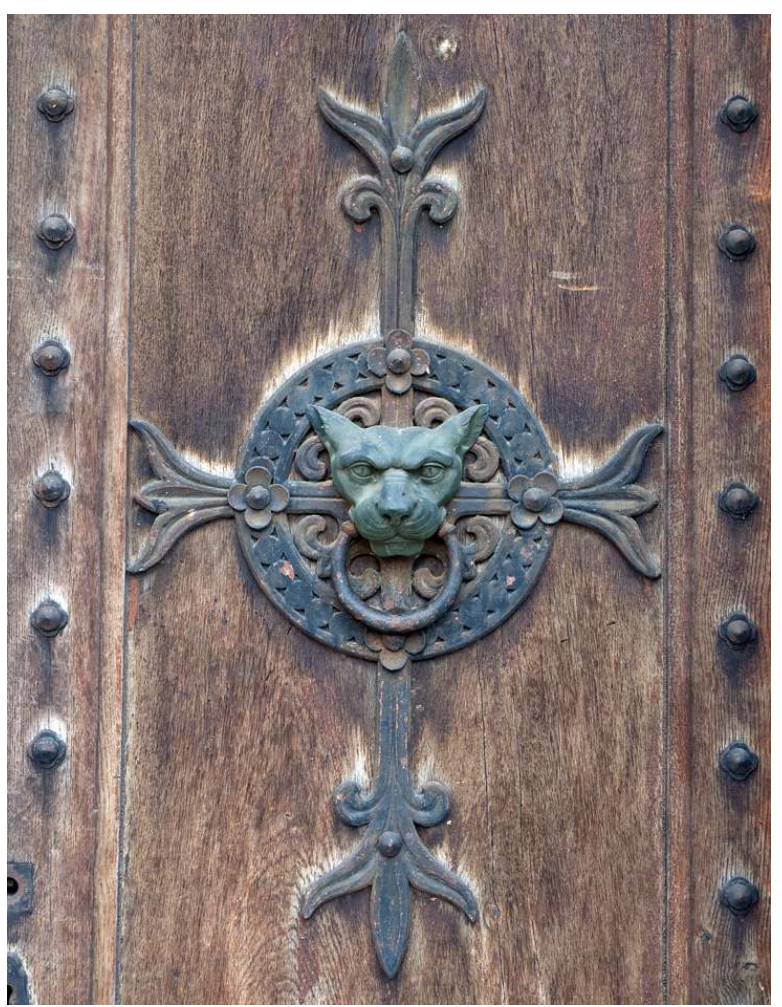

Heurtoir de la porte de l'église

Phot. C. Guégan, 2007. @ Inventaire du patrimoine culturel, Région Rhône-Alpes/Ville de Lyon, ADAGP

L'importance attachée par les visitandines à la clôture a sans doute dicté le parti architectural, au caractère presque défensif, de l'édifice. Il n'est pas anodin de noter que le terrain était bordé par les remparts de Saint-Just, et que pendant longtemps le seul accès depuis la rue s'est fait par une petite porte pratiquée dans ces derniers, ouvrant sur une sombre salle voûtée d'où partait une volée d'escalier conduisant à l'allée qui menait à l'entrée principale. Cet aspect de forteresse ${ }^{26}$ est également dû à l'utilisation de la pierre apparente, aux saillies des pavillons d'angle, en léger ressaut (fig. $\mathbf{n}^{\circ} \mathbf{6}$ ), aux ouvertures en forme de meurtrières sur l'avant-corps de l'élévation principale, aux vantaux de porte cloutés ornés de heurtoirs en forme de tête de lionne (fig. $\mathbf{n}^{\circ} \mathbf{7}$ ). S'affirment ici certaines particularités du style de Bossan, qui deviendront sa «marque de fabrique » : l'emploi du plein cintre et de la voûte en berceau, les élévations au soubassement nettement marqué, rythmées par des bandeaux en pierre de taille et de forts entablements, les fenêtres jumelées ou à meneaux, le goût pour les ferronneries très travaillées. Mais Bossan a su atténuer ce caractère austère par une certaine douceur dans le traitement des élévations, en usant de la polychromie (alternance de moellons en pierre jaune de Couzon, de brique rouge et de calcaire blanc pour la pierre de taille) et en animant les surfaces par de subtils décalages d'appareil. Les ouvertures sont soulignées d'un encadrement en bâtière en pierre de taille claire; au rez-de-chaussée, l'encadrement des baies en plein cintre est surmonté d'un rang de brique; un bandeau en pierre de taille court sur toutes les élévations de l'édifice, soulignant la séparation entre le rez-de-chaussée et les deux étages carrés ; la corniche, enfin, est rythmée par une frise de briques alternes en saillie sur trois rangs. Le chiffre trois, dont la symbolique est connue, se retrouve partout: triples baies jumelées aux angles de chaque étage, ainsi que sur les flancs sud et nord de l'église, 
ouverture en forme d'étoile de David (soit deux triangles embrassés) au sommet de la façade de l'église, cloître rythmé par des colonnes en saillie soutenant neuf arcades sur chacun de ses côtés. Une série de portes, dont le modèle a été fourni par Bossan, ouvre sur le cloître ; en chêne mouluré, elles sont entourées d'un encadrement à fronton en bâtière. Deux niches encadrées de briques agrémentent le mur de la galerie sud.

\section{L'aménagement intérieur}

Il reste aujourd'hui peu d'éléments du décor du couvent. Une grande partie du mobilier a suivi les visitandines dans leurs déménagements successifs, à Vaugneray puis à Annecy. D'autres ont été déposés par ces dernières soit au musée de Fourvière, soit au musée de la Visitation de Moulins ${ }^{27}$. Ce sont, encore une fois, les sources d'archives qui permettent de retracer l'aspect et l'évolution de ce décor, tant intérieur qu'extérieur. On sait ainsi que le chœur des religieuses contenait deux rangées de stalles de chêne foncé, apportées de la Croix-Rousse (aujourd'hui disparues) et était orné d'une statue de la Vierge, portant suspendu au cou un chapelet de nacre offert par le maréchal de Castellane, descendant de sainte Jeanne de Chantal, en $1850^{28}$. En 1858 fut mis en place un chemin de croix offert par leur aumônier et confesseur, l'abbé Cherbonnière ${ }^{29}$. Un grand tableau peint à l'huile, représentant « les saints fondateurs dans leur gloire, exécuté à Paris » était accroché (au plus tard en 1856) «au-dessus de la cheminée, dans (la) salle de communauté ${ }^{30}$. Les sources mentionnent également l'acquisition d'une niche d'exposition du SaintSacrement ${ }^{31}$, mais rien ne permet de préciser si elle se trouvait dans l'église ou dans le chœur des religieuses, ce qui est plus vraisemblable. En 1859, un autre chemin de croix fut érigé dans la chapelle des élèves ${ }^{32}$. Le 2 juillet de la même année, une statue de la Vierge prenait place dans la «salle d'ombrage » du pensionnat ${ }^{33}$. Brisée lors d'un orage, elle fut remplacée en 1860 par une statue de l'Immaculée Conception, dont les inscriptions gravées $\mathrm{du}$ socle, toujours en place dans la cour du pensionnat, correspondant à la description faite dans les Annales. Toutes les galeries du cloître étaient décorées de portraits de saints et de reliques ${ }^{34}$. Au pied de l'escalier principal se trouvaient deux statues de la Vierge et de saint Joseph, offertes en 1856 par Mme de Coton; deux autres statues de saint François et sainte Philomène étaient placées dans les niches de la galerie sud du cloître ${ }^{35}$. À la Noël 1859, une horloge aujourd'hui disparue était installée dans le clocher. Enfin le 1er 1861 mai, une statue de saint Joseph fut érigée au milieu du cloître, à l'emplacement d'une source qui venait d'y être découverte ${ }^{36}$. 
Figure 8

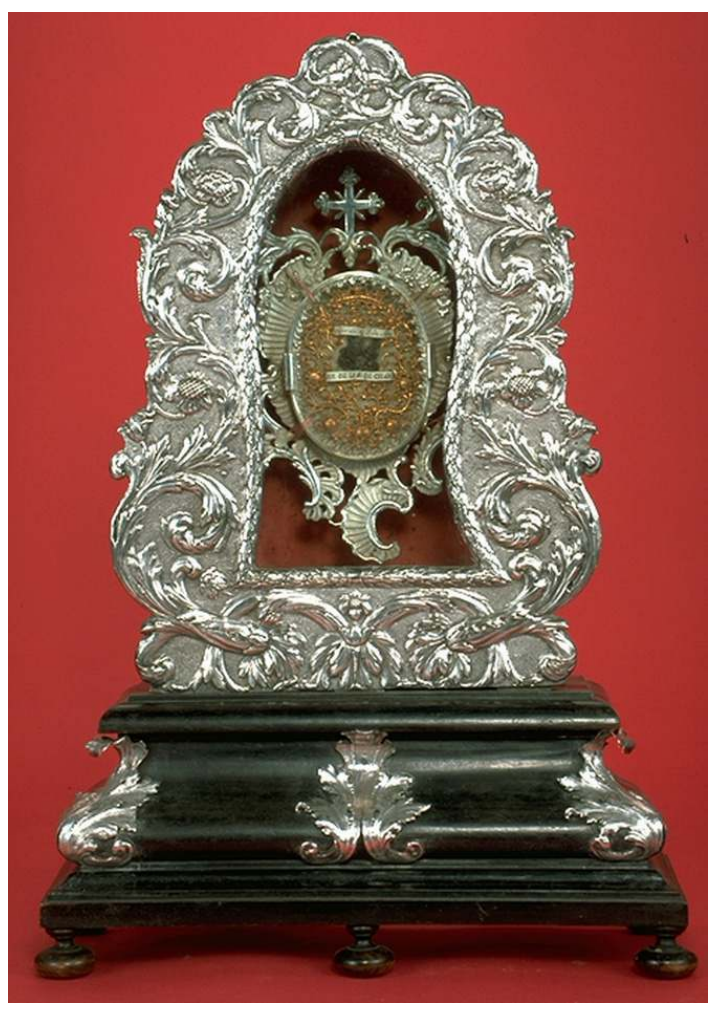

Monstrance contenant des reliques de saint François de Sales et sainte Jeanne de Chantal. Moulins, musée de la Visitation

(c) Musée de la Visitation - Moulins, 2007

Le Catalogue des Saintes Reliques, établi en $1914^{37}$, détaille un nombre impressionnant de reliques réparties dans tout le monastère. Dans le chœur des religieuses étaient conservées les plus précieuses, notamment celles des saints fondateurs : des fragments de la Vraie Croix et de la Sainte Épine, le lit de mort ${ }^{38}$ et les burettes d'argent ${ }^{39}$ de saint François de Sales, une urne contenant ses entrailles ${ }^{40}$, une monstrance (fig. $\mathbf{n}^{\circ} \mathbf{8}$ ) contenant des reliques de François de Sales et Jeanne de Chantal ${ }^{41}$. Y figuraient aussi des objets liés à l'histoire de l'ordre, notamment un portrait miniature de saint François de Sales et deux lettres de celui-ci, donnés par le cardinal de Bonald 29 janvier $1855^{42}$. Elles seront transférées en 1887 dans la salle du chapitre nouvellement créée. Peut-être est-ce à cette occasion que Mgr Déchelette fit don aux religieuses d'une statue de saint François de Sales (Petite Relation, p. 40) ? La pièce, dont les murs en plâtre gris cendré étaient ornés de sentences ${ }^{43}$, était décorée de boiseries de chêne clair à hauteur d'appui, comportait une chaire, des bancs faisant le tour de la salle, deux placards dans le mur et un meuble avec niche contenant l'effigie en cire de Anne-Madeleine Rémuzat, une visitandine du XVII ${ }^{\mathrm{e}}$ siècle étroitement liée à la dévotion au Sacré-Cœur. On y trouvait aussi un tabernacle avec un Christ en ivoire, donné par l'abbé Cherbonnière, un autel en bois polychrome et doré contenant une statue de saint Augustin ${ }^{44}$ et un tableau représentant Notre-Dame du Bon-Conseil. La chapelle des élèves n'était pas négligée. Grâce à leurs dons, elles purent commander à Bossan, en 1869, un autel de marbre blanc «orné de colombes argentées, de lettres formant le nom de Marie et d'une inscription latine signifiant «ton nom est une huile répandue " ${ }^{45}$. Cet autel fut vendu à une ancienne élève, Bénédicte Gonon, quand le pensionnat ferma en 1903. 


\section{L'église, laboratoire de formes}

L'aménagement et le décor intérieur de l'église (fig. $\mathbf{n}^{\circ}$ 9) ont été réalisés en deux temps. Après une interruption du chantier en 1857, date de l'achèvement du gros œuvre, les travaux reprirent en 1864, financés par différents dons. La marque de Bossan y est prédominante : il a en effet fourni des dessins pour l'ensemble du décor peint et sculpté et pour le mobilier, jusqu'aux éléments les plus modestes (ferrures du portail, pavement de mosaïque, barrière de communion). Sa richesse ornementale, qui contraste avec l'austérité de l'extérieur, constitue un élément particulier de l'œuvre. Elle répond autant au souhait des visitandines d'honorer la Vierge en concentrant dans le sanctuaire tous les fastes auxquels elles ont renoncé pour elles-mêmes ${ }^{46}$, qu'à la conception de Bossan sur l'ornementation. L'édifice est en effet pensé comme une œuvre d'art totale, subordonnant la peinture et la sculpture au monument en vue d'atteindre l'harmonie et l'unitée ${ }^{47}$. L'ornement prend ainsi une place fondamentale dans la création architecturale, au point que Bossan lui consacre la plus grande partie de son temps dans les années 1850-60 ${ }^{48}$. Ce décor, où s'imposent symbolisme végétal et animal liés à la Vierge et au Christ (fig. $\mathbf{n}^{\circ}{ }^{\circ}$ 10) , est construit selon une progression qui conduit l'œil du sol à la voûte. Bossan l'explique en ces termes : «Il faut établir dans l'ornementation une sorte de crescendo, en donnant aux parties supérieures le maximum de richesse - Voyez la plante; elle s'établit par les racines; la tige se développe avec simplicité, les richesses de la fleur brillante la couronnent $\aleph^{49}$. De fait, ce foisonnement décoratif recouvre l'entière surface des murs de la voûte et des parties saillantes de l'architecture, où les motifs, repris sur les éléments du mobilier, se répètent et se répondent. Il est rendu d'autant plus présent par l'utilisation d'une polychromie aux tonalités intenses, où prédominent l'azur, l'or et le rouge. Plus que la peinture historiée, ce sont donc les ornements symboliques et leur caractère répétitif qui confèrent à l'église sa monumentalité ${ }^{50}$. 
Figure 9

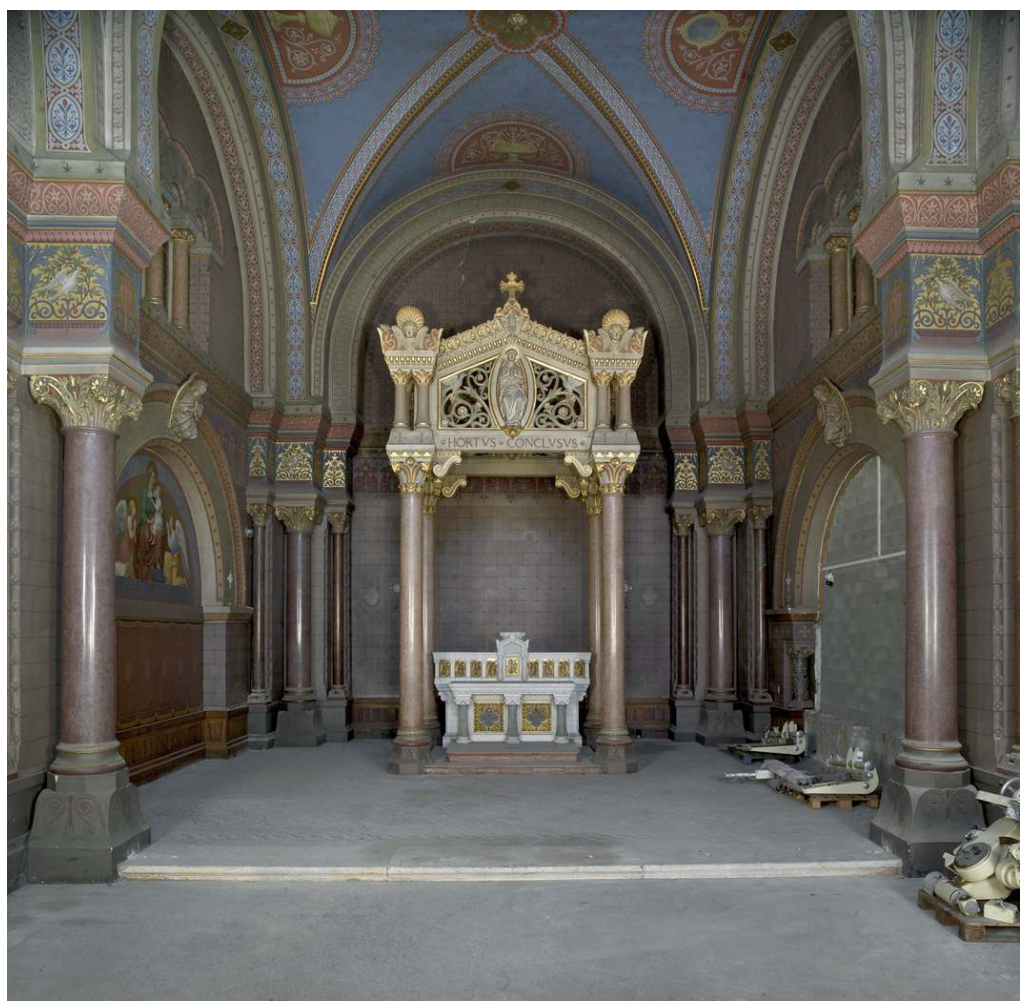

Vue d'ensemble de l'église, depuis la nef

Phot. É. Dessert, 2008. @ Inventaire du patrimoine culturel, Région Rhône-Alpes/Ville de Lyon, ADAGP 
Figure 10

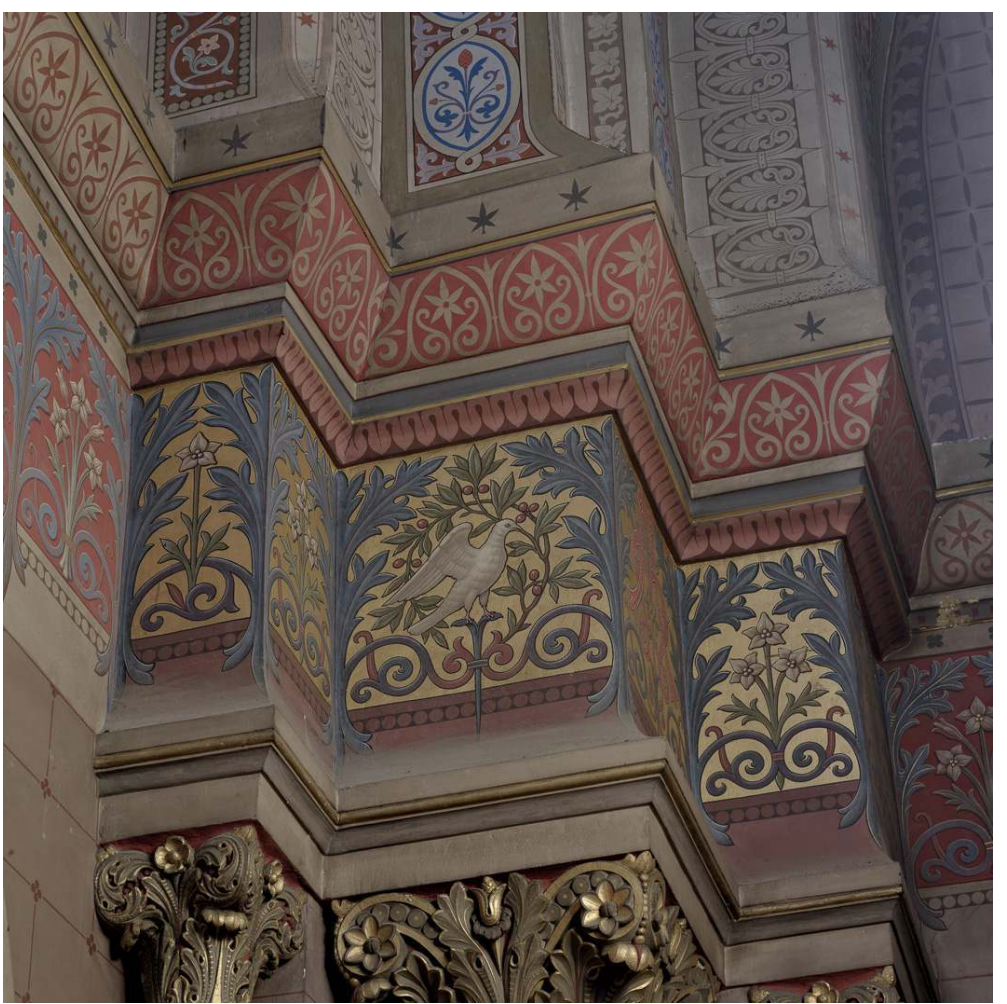

Église, détail de la frise d'entablement

Phot. É. Dessert, 2008. @ Inventaire du patrimoine culturel, Région Rhône-Alpes/Ville de Lyon, ADAGP 
Figure 11

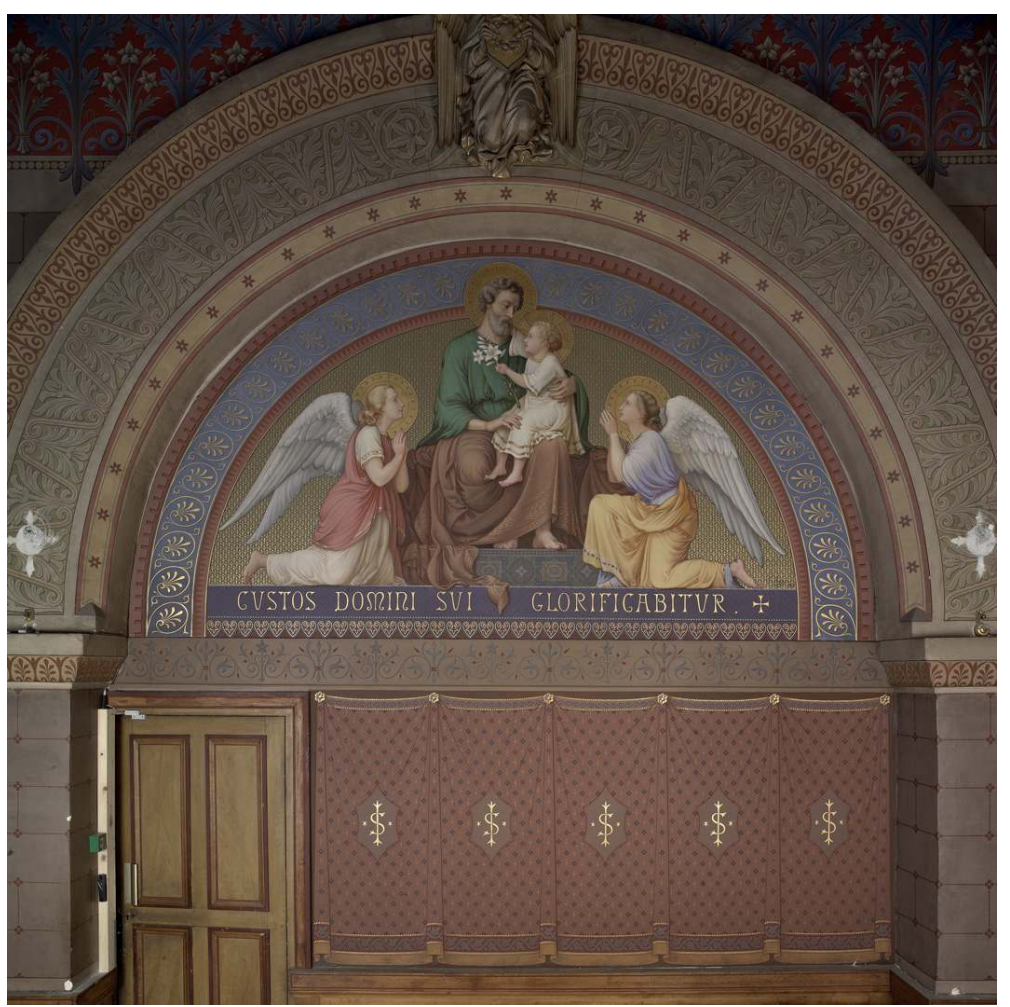

Claude-Baptiste Tailleur, Saint Joseph et l'Enfant entre deux anges, S.D.b.d.1864

Phot. É. Dessert, 2008. @ Inventaire du patrimoine culturel, Région Rhône-Alpes/Ville de Lyon, ADAGP

La décoration de l'église fut achevée début juillet 1865. Les sources sont peu prolixes sur les choix iconographiques; aucune ne mentionne le tableau de Claude-Baptiste Tailleur ${ }^{51}$, un élève de Janmot, Saint Joseph et l'Enfant entre deux anges ${ }^{52}$ (fig. $\mathbf{n}^{\circ} \mathbf{1 1}$ ), qui surmonte la porte de la sacristie donnant sur le chœur de l'église, à gauche du maître-autel, et fait face au chœur des religieuses, ni les motifs du décor peint ornant les parois et les voûtes. On sait cependant que Bossan préférait en confier l'exécution à de simples artisans plutôt qu'à des artistes de renom. Les archives du couvent n'ont pas révélé leur nom ${ }^{53}$. Cependant peintures et sculptures présentent de fortes parentés stylistiques avec celles réalisées plus tard pour la chapelle des dominicains d'Oullins ou pour l'église d'Ars. On peut ainsi penser qu'il fut exécuté par les mêmes artistes: Charles Dufraine, qui sera par la suite professeur à l'École des Beaux-Arts, pour les parties sculptées et Jacques Razuret pour les peintures décoratives. Ce que confirme, pour Dufraine, le Dictionnaire des artistes et ouvriers d'art du Lyonnais d'Audin et Vial ${ }^{54}$. Le maître-autel (fig. $\mathbf{n}^{\circ}{ }^{12}$ ), reproduit dans la monographie que Félix Thiollier a consacrée à Bossan ${ }^{55}$, témoigne de la richesse d'invention de l'architecte ${ }^{56}$. Il est surmonté d'un ciborium monumental, dressé sur des colonnes stuc imitant marbre rose et supportant un fronton en bâtière au tympan ajouré dans lequel sont représentés, sur la face antérieure, une Vierge dans une mandorle (fig. $\mathbf{n}^{\circ}$ 13), et sur la face postérieure, un agneau pascal. 
Figure 12

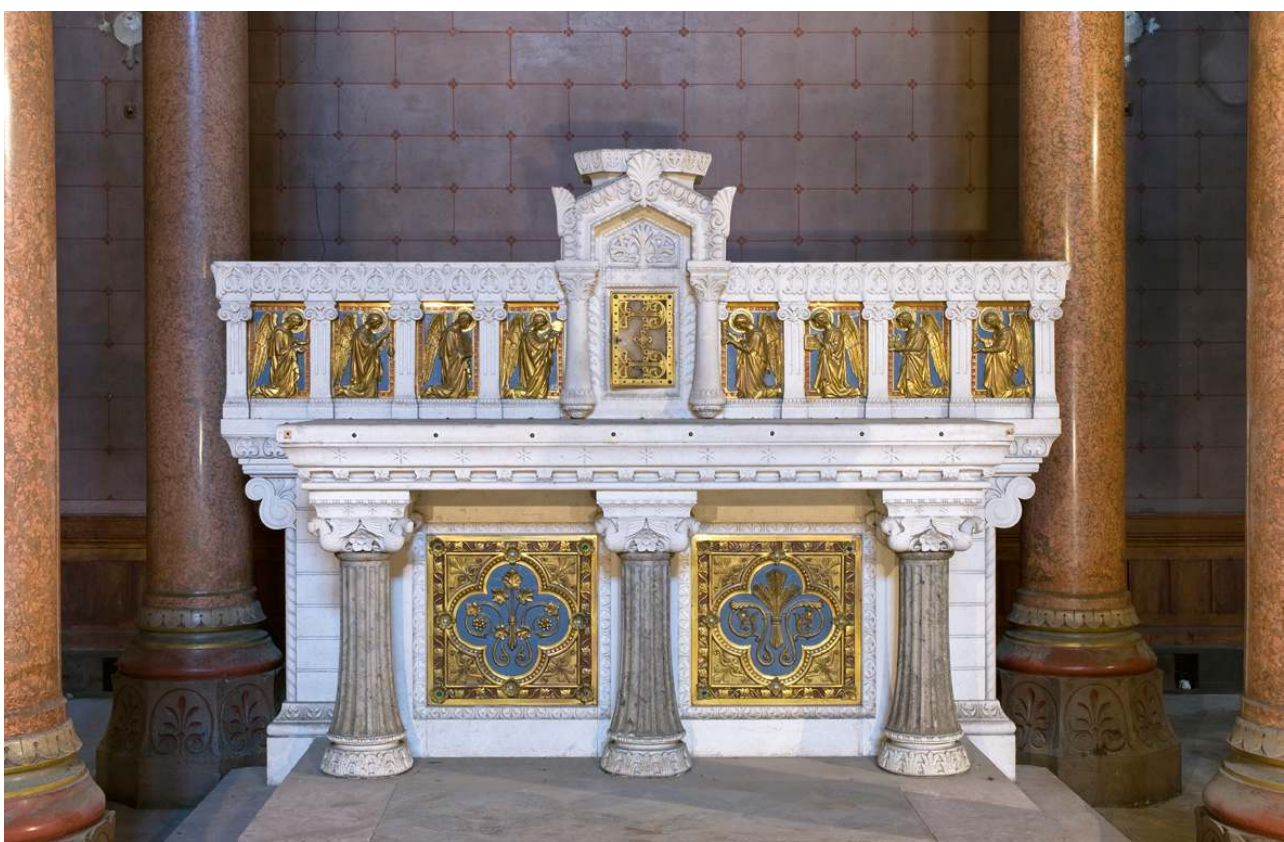

Église, maître-autel

Phot. C. Guégan, 2007. @ Inventaire du patrimoine culturel, Région Rhône-Alpes/Ville de Lyon, ADAGP

Figure 13

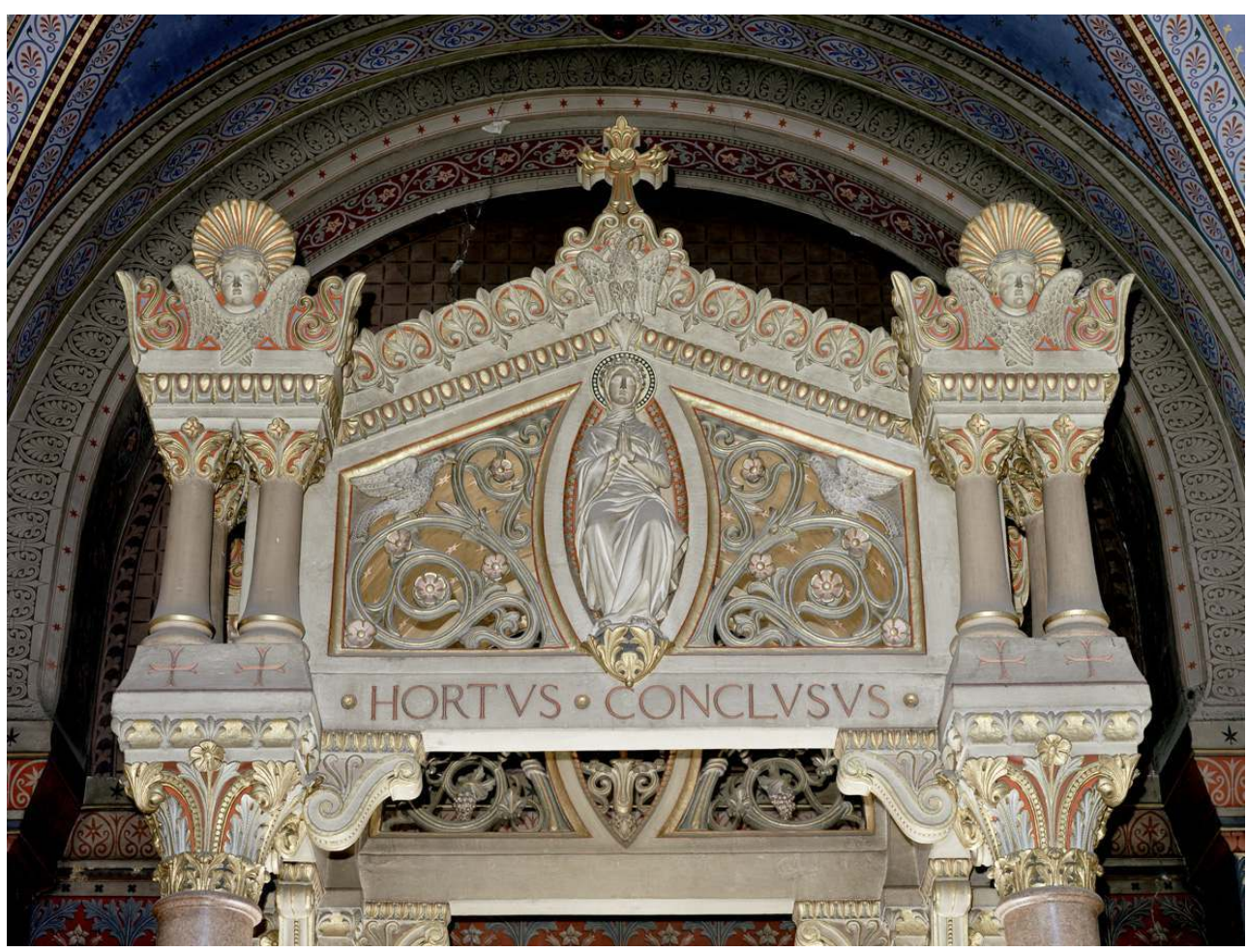

Église, ciborium, détail du tympan

Phot. É. Dessert, 2008. @ Inventaire du patrimoine culturel, Région Rhône-Alpes/Ville de Lyon, ADAGP 
Sur chacun des voûtains sont peints des symboles tirés des litanies de la Vierge ${ }^{57}$ (fig. $\mathbf{n}$ - 14) : tour de David, tour d'ivoire, temple du vrai Salomon, arche de la nouvelle alliance (dans la nef), vase précieux et porte du ciel (dans le chœur), complétés par un ciboire et un ostensoir dans l'axe du chœur des religieuses.

\section{Figure 14}

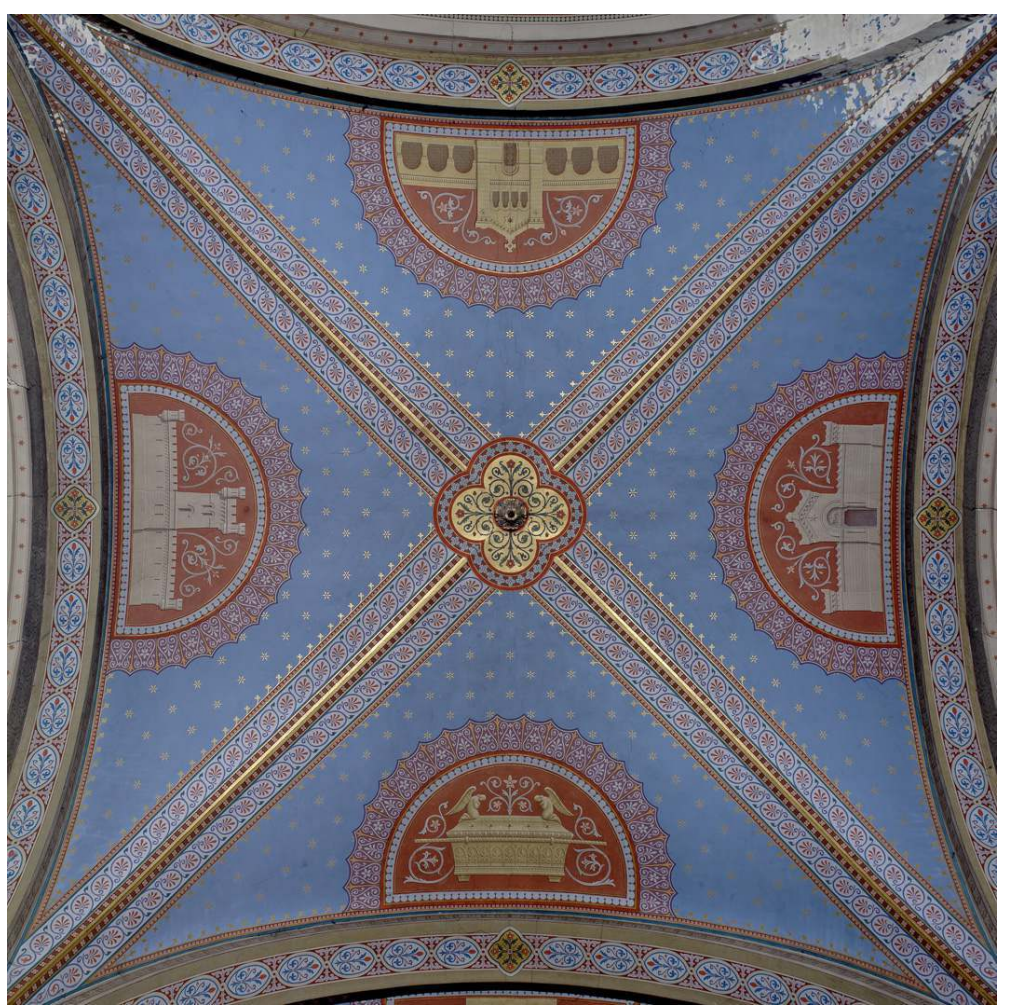

Église, décor de la voûte de la nef

Phot. É. Dessert, 2007. @ Inventaire du patrimoine culturel, Région Rhône-Alpes/Ville de Lyon, ADAGP

Dans un deuxième temps, en 1874, deux autels furent érigés dans la nef à l'initiative de la supérieure, Mère Marie-Régis Deville, l'un voué à saint François de Sales et l'autre au Sacré-Cœur. Ils sont surmontés de leurs statues respectives, placées dans une niche richement sculptée, dont le style rappelle celui de Dufraine. À cette date, on peut considérer le décor de l'église comme achevé. Le seul changement notable sera, en 1896, le remplacement de la cloche qui avait été apportée du monastère de la Croix-Rousse, par une nouvelle, baptisée par Mgr Déchelette.

\section{L'état actuel}

20 À partir du début du $20^{\mathrm{e}}$, le couvent entame un lent déclin; seul des travaux d'entretien ou de modernisation furent réalisés. En effet, après la fermeture du pensionnat en 1903, conséquence de la laïcisation de l'enseignement, la question financière devint omniprésente. Craignant la dissolution de l'ordre et leur dispersion, les visitandines se débarrassèrent du matériel scolaire et vendirent une partie de leur mobilier à d'anciennes élèves, se séparant de biens parfois précieux ${ }^{58}$. La guerre de 14-18 accrut leurs difficultés. Elles se virent obligées de louer les appartements de l'aumônerie, 
songèrent à se séparer du « bâtiment de la galerie », qui sera loué à partir d'octobre 1940 à des réfugiés venus de la région parisienne. C'est à cette date qu'est pratiquée une ouverture dans le mur coté rue du Juge de Paix (actuelle rue Roger-Radisson), destinée à ces nouveaux locataires et à préserver leur clôture. Vivant de plus en plus difficilement après 1945, elles décident en 1965 de vendre la totalité des bâtiments à la ville de Lyon, alors que prend forme le projet Malraux-Pradel de créer sur le site du couvent une école d'architecture et d'en utiliser l'église comme lieu de culte pour les habitants du quartier. Les visitandines continueront cependant d'occuper les lieux jusqu'à leur déménagement pour le couvent qu'elles ont fait construire à Vaugneray, où elles s'installent avec leurs sœurs de Condrieu en juin $1968^{59}$. Renonçant à son projet initial après 68 , la Ville fait néanmoins rénover le couvent, qu'elle loue en 1970 aux Hospices civils ${ }^{60}$, ces derniers souhaitant y loger des élèves infirmières. La configuration générale des bâtiments n'est pas modifiée : les travaux se restreignent à la réfection des toitures, au blanchiment des cellules et des couloirs et à l'installation de sanitaires et de mobilier.

En 1974, en raison de l'engorgement de leurs archives alors conservées à l'Hôtel-Dieu, les Hospices civils décident de transférer la totalité de leurs fonds à Fourvière. Les travaux, confiés à l'architecte en chef des Monuments historiques, Jean-Gabriel Mortamet (1930- ), s'étalent sur plusieurs années et affectent de manière plus profonde le bâtiment: des cloisons sont abattues, des ouvertures murées, des pièces entresolées. Le chœur des religieuses a totalement disparu dans ces aménagements on n'en perçoit plus aujourd'hui ni la fonction, ni les volumes. Certaines interventions « lourdes » ne seront cependant pas réalisées, les salles du rez-de-chaussée accueillant déjà des fonds d'archives ne pouvant être déplacés ${ }^{11}$. Enfin certaines zones n'ont jamais été rénovées, telles les cellules et couloirs du deuxième étages, ou les sacristies, néanmoins dépouillées de tout mobilier et en partie mutilées (dépose de l'escalier de la sacristie extérieure desservant la pièce du premier étage réservée au desservant, dépose du tour pour le passage des vases).

L'église, qui avait été peu touchée lors des premiers travaux d'aménagement du couvent, a davantage souffert par la suite. Perdant toute fonction cultuelle, elle a peu à peu été dépouillée de son mobilier : la barrière de communion et de la grille de clôture du chœur des religieuses ont été démontées, le chemin de croix déposé au musée des Hospices civils. Restés en place, les deux autels latéraux de la nef ont été partiellement démontés. Leurs tabernacles et des plaques de parement de marbre des gradins sont à l'heure actuelle entreposés dans un placard de la sacristie intérieure. L'absence d'entretien affecte par ailleurs la conservation du décor : les vitraux ont souffert de casses multiples, on relève d'importantes fissures au revers de l'élévation principale, et de nombreuses altérations des peintures murales à la retombée des voûtes et sur le mur sud (soulèvements, efflorescences de sels, perte de matière). Enfin, l'encrassement généralisé nuit à la perception de la richesse des couleurs et de l'ornementation, voulue par Bossan.

On ne peut aujourd'hui que souhaiter une restauration de l'ensemble des bâtiments, et particulièrement de l'église, qui en constitue la partie la mieux préservée. Elle permettrait, en les rendant accessibles au public, de redécouvrir un des jalons importants de la carrière de Bossan, dont la richesse est aujourd'hui ignorée de la plupart des lyonnais. À proximité immédiate de la basilique de Fourvière ${ }^{62}$, surplombant l'église Saint-Georges ${ }^{63}$, elle s'inscrit dans le cheminement à la fois artistique et spirituel autour duquel l'architecte a bâti son œuvre. 


\section{Annexe : œuvres de Bossan protégées au titre des Monuments historiques}

\section{Édifices religieux}

Église Saint-Georges (Lyon 5e), 1844 : inscrite, arrêté du 29.12.1982

Église de Couzon au Mont-d'Or, Rhône, 1855 : clocher inscrit, arrêté du 5.7.1927

Église Notre-Dame de Feurs (Loire), 1861 : inscrite, arrêté du 19.11.1991 ; Bossan est l'auteur de la façade et du clocher

Basilique d'Ars-sur-Formans (Ain), 1862 : classée, arrêté du 2.2.1982

Basilique de Fourvière (Lyon 5e), 1872-1896 : inscrite, arrêté du 26.9.1977

Église Notre-Dame-du-Rosaire du couvent Saint-Lazare, dite église des dominicains (Marseille 6e), 1878 : inscrite, arrêté du 28.9.1995.

\section{Objets mobiliers et immeubles par destination dont Bossan a fourni les modèles}

Collégiale Notre-Dame-de-l'Espérance, Montbrison (Loire) :

- tribune d'orgue, vantaux de la porte intérieure, 1840 : classés, liste de 1840

- stalles du chœur, 1865 : classées, arrêté du 30.12.1982

Primatiale Saint-Jean (Lyon 5e) :

- stalle épiscopale, 1845 : classée, arrêté du 29.01.1982

- chasuble, s.d. : classée, arrêté du 7.12.1989

Église Saint-Polycarpe (Lyon 1er) :

- autel et retable de la Vierge, 1852 ; chaire, 1864 : classés, arrêté du 11.07.1978

Église de l'Immaculée-Conception (Lyon 6e) :

- ostensoir (réalisé par Armand-Caillat), 1866 : classé, arrêté du 26.11.1993

Église Saint-François-de-Sales (Lyon 2e) :

- ostensoir (réalisé par Armand-Caillat), 1885 : classé, arrêté du 18.02.1999

Église paroissiale Saint-Pierre, Mornant (Rhône) : 
- stalles, lambris, maître autel, autel de la chapelle de la Vierge, tribune des pénitents, fonts baptismaux, porte-cierges votif, chandeliers d'autel, 1854-59 : classés, arrêté du 3.9.1979.

\section{NOTES}

1. Voir dans la : base Mérimée : notice PA69000023.

2. La principale étude existant actuellement est celle de Chazot, Marie. Histoire du Monastère de la Visitation Sainte-Marie de Lyon à Fourvière, Maîtrise d'histoire contemporaine, sous la direction de Nathalie Mathian, Université de Lyon III, 2002. Une partie des recherches liées à la présente étude a été réalisée par Gaëlle Le Page, stagiaire au Service régional de l'Inventaire du patrimoine culturel d'avril à juin 2007.

3. Archives privées Visitation Annecy. Sans cote. Acte de vente de la propriété de M. JeanPierre Garcin, drapier, aux dames de la Visitation de la Croix-Rousse et du Carmel, 10 octobre 1850.

4. Gymnase en 1870, construction perpendiculaire à l'aile sud désapprouvée par Bossan, «bâtiment de la galerie » destiné au pensionnat en 1890, édifié sur le fond nord-ouest de la parcelle par Sainte-Marie Perrin, aux accents de Renaissance florentine, aumônerie en 1890 par la surélévation d'un étage du gymnase.

5. La construction fut en grande partie financée par des emprunts et des dons d'autres établissements religieux, (Petite Relation de notre transfert de la Croix Rousse à Fourvière, AP Visitation Annecy, ms. sans cote, 1896 ca., p. 4).

6. Dufieux, Philippe. Le mythe de la primatie des Gaules. Pierre Bossan et l'architecture religieuse en Lyonnais au XIX ${ }^{e}$ siècle. Lyon : P.U.L., 2004, p. 89.

7. AP Visitation Annecy, Annales, 1840-55, p. 323.

8. AP Visitation Annecy, Annales, 1840-55, p. 375.

9. La vente du couvent de la Croix-Rousse ne fut réalisée qu'en 1855 .

10. Archives communales Lyon, 315 WP 96 ; AP Visitation Annecy, Annales 1840-55, p. 262.

11. Dufieux, Philippe. Le mythe de la primatie des Gaules. Pierre Bossan et l'architecture religieuse en Lyonnais au XIX ${ }^{e}$ siècle. Lyon : P.U.L., 2004, p. 295.

12. AP Visitation Annecy, Annales, 1840-55, p. 262.

13. Charvet, Léon. Lyon artistique. Architectes : notices biographiques et bibliographiques avec une table des édifices et la liste chronologique des noms. Lyon: 1899, p. 157-158 et p. 219-220.

14. Petite Relation de notre transfert de la Croix Rousse à Fourvière, AP Visitation Annecy, ms. sans cote, 1896 ca., p. 10-11.

15. Petite Relation de notre transfert de la Croix Rousse à Fourvière, AP Visitation Annecy, ms. sans cote, 1896 ca., p. 16.

16. Les quatre ailes du bâtiment et l'église sont légèrement désaxées par rapport aux points cardinaux. Ainsi l'aile désignée comme nord est en fait orientée nord-nord-est, l'aile sud, sudsud-ouest, etc. Pour des raisons de commodité dans la description, nous avons choisi de les désigner par le point cardinal dont elles se rapprochaient le plus.

17. Jean, $6,51$.

18. Proverbes, 8,31 .

19. AP Visitation Annecy, Annales, $1855-70$, p. 42-44.

20. Petite Relation de notre transfert de la Croix Rousse à Fourvière, AP Visitation Annecy, ms. sans cote, 1896 ca., p. 14.

21. AP Visitation Annecy, Annales, 1880-99, p. 319. 
22. AP Visitation Annecy, Annales, 1880-99, p. 366.

23. AP Visitation Annecy, Annales, 1899-1923, p. 408.

24. AP Visitation Annecy, Annales, 1855-70, p. 118.

25. AP Visitation Annecy, Annales, 1855-70, p. 42-44.

26. Petite Relation de notre transfert de la Croix Rousse à Fourvière, AP Visitation Annecy, ms. sans cote, 1896 ca., p. 13 : « Notre nouvelle demeure qui occupe, comme nous l'avons dit, le point culminant de la colline, est loin de déparer ce paysage. Elle présente un peu l'aspect d'un château-fort, de style roman ».

27. Un certain nombre d'entre eux ont été présentés à l'exposition Splendeurs dévoilées. Cinq siècles d'art à la Visitation, Moulins, 2007, dont le catalogue donne un historique et une description très détaillés.

28. Petite Relation de notre transfert de la Croix Rousse à Fourvière, AP Visitation Annecy, ms. sans cote, 1896 ca., p. 3.

29. Petite Relation de notre transfert de la Croix Rousse à Fourvière, AP Visitation Annecy, ms. sans cote, 1896 ca., p. 17 ; Annales 1840-80, p. 341. À sa mort en 1861, une plaque (toujours en place) vint sceller une cavité destinée à recevoir son cœur, à l'arrière du maître autel de l'église.

30. Petite Relation de notre transfert de la Croix Rousse à Fourvière, AP Visitation Annecy, ms. sans cote, 1896 ca., p. 16.

31. Petite Relation de notre transfert de la Croix Rousse à Fourvière, AP Visitation Annecy, ms. sans cote, 1896 ca., p. 21.

32. AP Visitation Annecy, Annales, 1855-70, p. 346.

33. AP Visitation Annecy, Annales, 1855-70, p. 104.

34. AP Visitation Annecy, Catalogue des saintes reliques, 1914; le Coutumier de l'ordre préconisait de mettre « au bout des allées du cloître, des tableaux ou de grandes images dévotes, et au lieu le plus convenable, un petit autel de 3 pieds et demi devant lequel on s'arrêtera en procession » (éd. 1850, p. 144).

35. AP Visitation Annecy, Annales, 1855-70, p. 42-44.

36. Petite Relation de notre transfert de la Croix Rousse à Fourvière, AP Visitation Annecy, ms. sans cote, 1896 ca., p. 17.

37. AP Visitation Annecy. Sans cote.

38. Aujourd'hui conservé au musée de Fourvière ; à Sainte-Marie-de-Bellecour jusqu'en 1792, il fut déposé à cette date chez la comtesse de la Barmondière, puis donné par cette dernière à la communauté de la Croix-Rousse le 20 octobre 1841.

39. Musée de la Visitation, Moulins.

40. Cette urne a été emportée en exil par les sœurs de Lyon, d'abord à Venise, puis à Trévise (Archives diocésaines, Lyon, 3 III 52, in Chazot, Marie. Histoire du Monastère de la Visitation Sainte-Marie de Lyon à Fourvière, Maîtrise d'histoire contemporaine, sous la direction de Nathalie Mathian, Université de Lyon III, 2002, p. 23).

41. Chazot, Marie. Histoire du Monastère de la Visitation Sainte-Marie de Lyon à Fourvière, Maîtrise d'histoire contemporaine, sous la direction de Nathalie Mathian, Université de Lyon III, 2002, p. 2 ; œuvre de Louis Catinet, orfèvre à Lyon, vers 1714-15; apporté à Lyon par les religieuses de Châlons, après la Révolution. Modifié en 1751 : ajout d'un reliquaire d'argent au décor auriculaire et d'un gant de cérémonie de saint François de Sales; déposé à Moulins par le monastère de Scy-Chazelle en 1999 ; repr. in cat. exp. Moulins, 2007, nº 59, p. 123.

42. AP Visitation Annecy, Annales, 1855-70, p. 4 ; Petite Relation de notre transfert de la Croix Rousse à Fourvière, AP Visitation Annecy, ms. sans cote, 1896 ca., p. 9.

43. AP Visitation Annecy, Annales, 1880-99, p. 149.

44. Était au couvent de Vaugneray ; localisation actuelle inconnue. 
45. AP Visitation Annecy, Annales, 1855-70, p. 594 ; Chazot, Marie. Histoire du Monastère de la Visitation Sainte-Marie de Lyon à Fourvière, Maîtrise d'histoire contemporaine, sous la direction de Nathalie Mathian, Université de Lyon III, 2002, p. 82.

46. Chazot, Marie. Histoire du Monastère de la Visitation Sainte-Marie de Lyon à Fourvière, Maîtrise d'histoire contemporaine, sous la direction de Nathalie Mathian, Université de Lyon III, 2002, p. 248 : «Pour que ce bijou de chapelle soit vraiment comme une épouse parée pour son époux, il faut que la main du peintre la parcoure de la base à la voûte ».

47. Dufieux, Philippe. Le mythe de la primatie des Gaules. Pierre Bossan et l'architecture religieuse en Lyonnais au XIX ${ }^{\mathrm{e}}$ siècle. Lyon : P.U.L., 2004, p. 82.

48. Thiollier en a publié un grand nombre sous forme de planches gravées à la fin de la monographie qu'il a consacrée à Bossan: Thiollier, Félix. L'Euvre de Pierre Bossan, architecte : basiliques, églises, chapelles, monuments civils, tombeaux, bronzes, orfèvrerie, etc. Monographie de la chapelle de Saint-Thomas d'Aquin à Oullins (Rhône), peintures, sculptures, décoration intérieure, par P. Borel, C. Dufrayne, J. Razuret. Montbrison : 1891.

49. Thiollier, Félix. L'Euvre de Pierre Bossan, architecte : basiliques, églises, chapelles, monuments civils, tombeaux, bronzes, orfèvrerie, etc. Monographie de la chapelle de Saint-Thomas d'Aquin à Oullins (Rhône), peintures, sculptures, décoration intérieure, par P. Borel, C. Dufrayne, J. Razuret. Montbrison : 1891, p. 21.

50. Dufieux, Philippe. Le mythe de la primatie des Gaules. Pierre Bossan et l'architecture religieuse en Lyonnais au XIX ${ }^{\mathrm{e}}$ siècle. Lyon : P.U.L., 2004, p. 257.

51. Né à Besançon en 1823, actif à Lyon entre 1860 et 1871 (Audin, Marius, Vial, Eugène. Dictionnaire des artistes et ouvriers d'art de la France, Lyonnais. Paris, 1918, t. 2, p. 249).

52. Signé et daté 1864 .

53. De même qu'elles ne mentionnent pas la date d'acquisition et de mise en place du chemin de croix, déposé et conservé au musée des Hospices civils.

54. T.1, p. 302.

55. Thiollier, Félix. L'Euvre de Pierre Bossan, architecte : basiliques, églises, chapelles, monuments civils, tombeaux, bronzes, orfèvrerie, etc. Monographie de la chapelle de Saint-Thomas d'Aquin à Oullins (Rhône), peintures, sculptures, décoration intérieure, par P. Borel, C. Dufrayne, J. Razuret. Montbrison : 1891, p. 82, nº 23.

56. Il vint remplacer un autel plus ancien, peut-être rapporté de la Croix-Rousse, qui fut donné au couvent de Condrieu (AP Visitation Annecy, Annales, 1855-70, p. 247-48).

57. Chazot, Marie. Histoire du Monastère de la Visitation Sainte-Marie de Lyon à Fourvière, Maîtrise d'histoire contemporaine, sous la direction de Nathalie Mathian, Université de Lyon III, 2002 , p. 65.

58. Les " antiques armoires des archives" et l'autel de la chapelle des élèves notamment. AP Visitation Annecy, Annales, 1900-1923, p. 155 ; Chazot, Marie. Histoire du Monastère de la Visitation Sainte-Marie de Lyon à Fourvière, Maîtrise d'histoire contemporaine, sous la direction de Nathalie Mathian, Université de Lyon III, 2002, p. 92.

59. A. diocésaines, Lyon, 3 III 52 (1962-68) ; AP Visitation Annecy, AV 65-68.

60. Ils en deviendront propriétaires en 1978, à la suite d'un échange de terrains à Gerland avec la ville.

61. Chazot, Marie. Histoire du Monastère de la Visitation Sainte-Marie de Lyon à Fourvière, Maîtrise d'histoire contemporaine, sous la direction de Nathalie Mathian, Université de Lyon III, 2002, p. 102.

62. Voir dans la base Mérimée : notice PA00117783.

63. Voir dans la base Mérimée : notice PA00117797. 


\section{RÉSUMÉS}

Propriété des Hospices civils de Lyon, l'ancien couvent de la Visitation Sainte-Marie de Fourvière est l'une des premières œuvres lyonnaises de Pierre-Marie Bossan. Sa transformation en dépôt d'archives au milieu des années 1970 a entraîné d'importantes modifications de sa structure. À partir de sources anciennes et de rares études récentes, cet article se propose de retracer les principales étapes de sa construction et de son aménagement intérieur, en insistant plus particulièrement sur le décor de l'église. C'est en effet là que s'affirment déjà dans leur plénitude les principaux éléments du vocabulaire ornemental de l'architecte, dont la théorie sera transcrite à la fin du XIX ${ }^{\mathrm{e}}$ siècle par son premier biographe, Félix Thiollier.

Property of the Hospices civils of Lyon, the former convent of the Visitation Sainte-Marie de Fourvière is one of the first works of Pierre-Marie Bossan in Lyon. Its transformation in warehouse for their archives in the middle of 1970s led to important modifications of its structure. From ancient sources and from rare recent studies, this article suggests redrawing the main stages of its construction and its internal development, by insisting more particularly on the decoration of the church. It is indeed there that already assert themselves in their plenitude the main elements of the decorative vocabulary of the architect, the theory of which will be transcribed at the end of the 19th century by his first biographer, Félix Thiollier.

\section{INDEX}

Mots-clés : service central des archives des Hospices civils de Lyon, Charles Dufraine, MarieAimée Bossan, Mère Marie-Régis Deville, Mgr Franzoni, cardinal de Bonald, abbé Cherbonnière, Mgr Déchelette, Anne-Madeleine Rémuzat, maréc hal de Castellane, Mme de Coton, Bénédicte Gonon, hôpital mère-enfant de Bron, hôpital Édouard-Herriot, l'Antiquaille, Debrousse, PierreMarie Bossan, Clément Martinant, Charles Franchet, Guillaume Léo, Sainte-Marie Perrin, JeanGabriel Mortamet, Claude-Baptiste Tailleur, Janmot, Jacques Razuret, couvent de visitandines Lyon, couvent de carmélites Lyon, chapelle des enfants de Marie, couvent de visitandines Vaugneray, couvent de visitandines Annecy, chapelle des dominicains d'Oullins, église d'Ars, basilique de Fourvière Lyon, église Saint-Georges Lyon, musée de Fourvière, musée de la Visitation de Moulins, vente du site de Fourvière, reconversion du site de Fourvière, colline de la Croix-Rousse, remparts de Saint-Just, Hospices civils de Lyon, couvent de la Visitation SainteMarie de Fourvière Lyon, couvent de Bellecour Lyon, couvent de l'Antiquaille Lyon

\section{AUTEUR}

\section{CATHERINE GUÉGAN}

Conservateur du patrimoine, Service de l'inventaire du patrimoine culturel, Région Rhône-Alpes. cguegan@rhonealpes.fr 